\title{
Design of a Missile-borne Conformal Microstrip Navigation Antenna
}

\author{
Junyan $\mathrm{Wu}^{1}$, Jiaao $\mathrm{Yu}^{1}$ and Qin $\mathrm{Tao}^{2}$ \\ ${ }^{1}$ Air Force Early Warning Academy, Wuhan, 430019, China \\ ${ }^{2}$ PLA, No.94639 Troop, Nanjing 211599, China
}

\begin{abstract}
A missile-borne conformal dual-fed circular polarized microstrip antenna array is designed and fabricated, which realized omnidirectional radiation in horizontal direction. Frequencies of the antenna include GPS L1 frequency (1.575GHz) and Beidou navigation system B1 frequency (1.562GHz). Based on the HFSS simulation, the influence of conformation is analyzed on $S_{11}$, axial ratio and gain of antenna array. The size of microstrip patches is changed so that the influence of conformation on antenna can be reduced. The circular polarization performance and the circular polarization gain of the antenna are also improved. The antenna array prototype is fabricated and measured. The result shows that the $S_{11}$ parameter of the antenna is less than $-10 \mathrm{~dB}$ in the $1.55 \sim 1.60 \mathrm{GHz}$ frequency band. At the GPS L1 frequency and the Beidou $\mathrm{B} 1$ frequency, the maximum of horizontal omnidirectional gain is $1.73 \mathrm{~dB}, 1.25 \mathrm{~dB}$ respectively. The out-of-roundness of the radiation pattern is less than $2.5 \mathrm{~dB}$. The antenna has a good horizontal omnidirectional circular polarization radiation performance.
\end{abstract}

\section{Introduction}

The conformal microstrip antenna based on the flexible thin medium can maintain the aerodynamic performance of the carrier without destroying the mechanical structure of the carrier, and can save the internal space of the carrier. This antenna can be made into a communication antenna or a navigation antenna mounted on the combat platforms such as guided missiles, ships and aircraft. The above advantages of conformal microstrip antennas have make it been widely used. Now it has become one of the hotspots in the field of antenna. In [1,2], the factors that affect the omnidirectional of antenna were analyzed, and a horizontal omnidirectional conformal microstrip antenna array was designed and fabricated. In [3], the problem of narrow bandwidth of microstrip antenna was studied, and a wideband technology of conformal microstrip antenna was proposed. In [4,5], methods for analyzing and calculating the conformal antenna on dielectric carrier were proposed. In [6-8], the electromagnetic band gap structure was introduced into the design of conformal microstrip antenna. The surface wave was suppressed, the influence of surface curvature on the antenna was reduced, and the radiation performance of the antenna was improved. In [9-11], the pattern synthesis of conformal microstrip antenna arrays was studied, and several fast algorithms with low mutual coupling and high accuracy was proposed.

Most of conformal microstrip antenna array adopt the design of directly conforming the planar antenna to the large curvature radius carrier. When the radius of curvature of the carrier is greater than one wavelength, the effect of curvature change on the microstrip antenna can be neglected. However, when the radius of curvature of the carrier is too small, the plane antenna model is no longer applicable. Patch bending will affect the bandwidth, gain and beam width of the antenna. In particular, it has a greater impact on the circular polarization performance and reduces the ability of the antenna to receive signals $[12,13]$.

The satellite navigation signals are mostly circular polarization signals operating in the $\mathrm{L}$ band. When the missile body is electrically small for navigation signals, the planar structure antennas are unable to be directly mounted on the surface of the missile. If the antenna is curved and conformed to the surface of the missile, the influence of the surface material and shape of the carrier on the performance of the antenna cannot be neglected. Especially, the influence of curvature radius on the circular polarization performance of antenna is more obvious. This will adversely affect the reception of navigation signals.

In this paper, a conformal omnidirectional circular polarization navigation antenna array on an electrically small missile body was designed by the way that the microstrip patch antenna was conformed on the circular truncated cone. The circular polarization gain was improved by adjusting the size of the antenna array element. In the end, the antenna was fabricated and tested. The measured results show that the conformal antenna has good circular polarized radiation performance at GPS L1 frequency points $(1.575 \mathrm{GHz})$ and Beidou B1 frequency points $(1.562 \mathrm{GHz})$. 


\section{Microstrip antenna design}

\subsection{Antenna element design}

The planar structure of the double-fed circularly polarized microstrip antenna element is shown in Figure 1. The antenna is based on the rectangular microstrip patch structure and operates on the GPS L1 frequency point. The width of the microstrip patch is $W$, the length is $L$, and the patch is printed on the flexible dielectric substrate with a relative dielectric constant of $\varepsilon_{r}=2.65$. The thickness of the substrate is $0.6 \mathrm{~mm}$. There are two input ports at the edge of the antenna, which are fed by two orthogonal signals respectively. Circular polarization radiation is excited by two orthogonal signals. The orthogonal signals are generated by Wilkinson power divider and phase shifter. The $a_{1}$ and $a_{2}$ belong to the Wilkinson power divider, which ensure the equal amplitude of the two signals. $a_{3}$ is a phase shifter to realize phase shifting function and ensure the orthogonality of two signals. The impedance of $a_{1}$, $a_{2}$ and $a_{3}$ is $Z_{1}=90 \Omega, Z_{2}=90 \sqrt{2} \Omega, Z_{3}=90 \Omega$, respectively. The corresponding linewidth is $w_{1}=0.53 \mathrm{~mm}, \quad w_{2}=0.2 \mathrm{~mm}, \quad w_{3}=0.53 \mathrm{~mm}$.

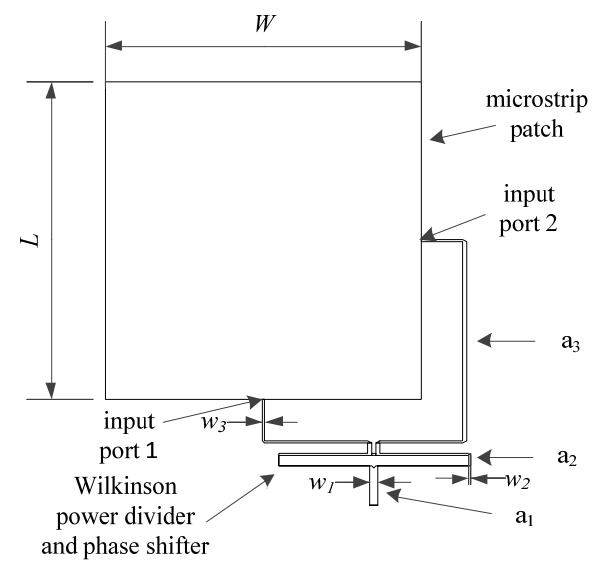

Figure 1. The plane structure of microstrip antenna unit

\subsection{Antenna array design}

The structure of the missile-borne conformal antenna array is shown in Figure 2. The antenna array is composed of three antenna elements. The antenna element is evenly distributed on the surface of the cone at the same distance. The three elements are fed by one-to-three power divider network, and the horizontal omnidirectional radiation is realized. The top diameter of the circular truncated cone is $D_{u p}=78.3 \mathrm{~mm}$, the bottom diameter is $D_{\text {down }}=113 \mathrm{~mm}$, and the height is $H=110 \mathrm{~mm}$.

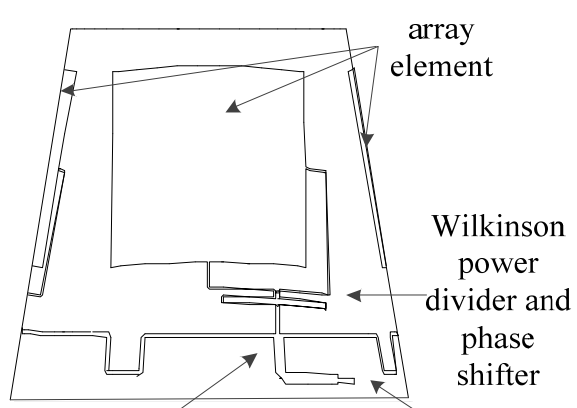

1-to-3 feed network $50 \Omega$ feed position

Figure 2. The structure of missile-borne conformal microstrip antenna array

The structure of one-to-three power divider network is shown in Figure 3. The one-to-three power divider network performs the power allocation while achieving the impedance transformation of one $50 \Omega$ impedance port to three $90 \Omega$ impedance ports. The $1 / 4$ wavelength impedance conversion section is added to the network, and the impedance of the transform section is $31.7 \Omega$. The width of the line is $w_{2}=2.34 \mathrm{~mm}$, and the length of the line is $y_{1}=11.3 \mathrm{~mm}$ and $y_{2}=21 \mathrm{~mm}$. At the end of it, the $50 \mathrm{ohm}$ microstrip transmission line with a width of $w_{3}=1.6 \mathrm{~mm}$ is connected, which match with the $50 \Omega$ SMA connector. The phase adjustment branch is added on the transmission line, so that port 3 and port 4 can be delayed by $2 \pi$ relative to the port 2 in phase, that is, the distance of one wavelength $\lambda_{g}=129 \mathrm{~mm}$. Thus ensuring port 2, 3 and 4 are equal amplitude and phase fed[14].

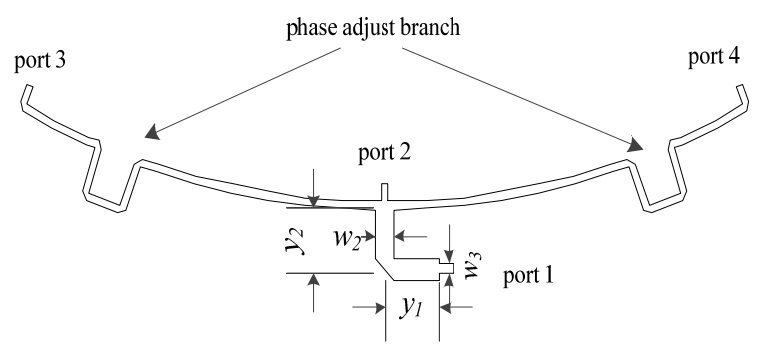

Figure 3. The structure of one-to-three power divider network

\section{Analysis of the influence of missile conformation on the antenna perfor- mance}

After design and adjustment, the planar microstrip antenna element resonances at $1.575 \mathrm{GHz}$ frequency point and has a good circularly polarized radiation performance. Now the antenna patch is square and the size of antenna is $W=L=58.7 \mathrm{~mm}$. The antenna gain curve and axial ratio curve obtained by simulation are shown in Figure 4. Then conform the antenna to the truncated cone and remain its dimension parameters unchanged. The variation of the antenna gain curve and the axial ratio curve is shown in Figure 4. The simulation results show that the gain maxima is almost unchanged after the antenna is conformed, but the optimal frequency 
of the gain is shifted from $1.575 \mathrm{GHz}$ to $1.583 \mathrm{GHz}$, as shown in Figure 4(a). After conformation, the axle ratio performance is worse. The optimal frequency of the axial ratio is shifted from $1.575 \mathrm{GHz}$ to $1.59 \mathrm{GHz}$, which deviates from the resonant frequency of the antenna, as shown in Figure 4(b).

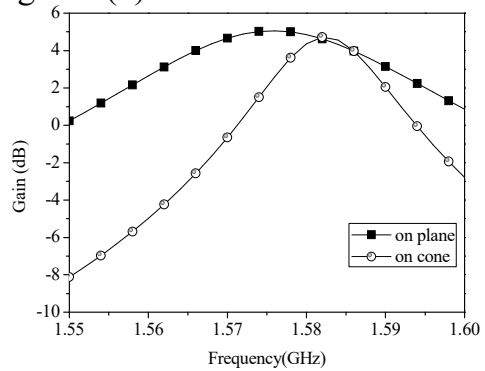

(a) Gain of the antenna

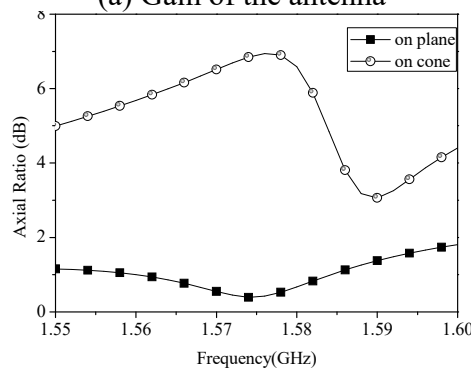

(b) Axial ratio of the antenna

Figure 4. Performance comparison of planar antenna and conformal antenna

In order to further analyze the cause of antenna performance change, we simulated the conformal antenna as a two port network. The position of the antenna input port 1 and 2 is shown in Figure 1. The input impedance curves of the two input ports are obtained by simulation, as shown in Figure 5. After antenna conformation, the input impedance of port 2 changes significantly compared to port 1 . The change of impedance's real part will lead to different signal amplitude. The change of impedance's imaginary part will lead to different signal phases [15]. The equal amplitude and orthogonality of the two signals cannot be ensured, which makes the circular polarization performance of the antenna deteriorate.

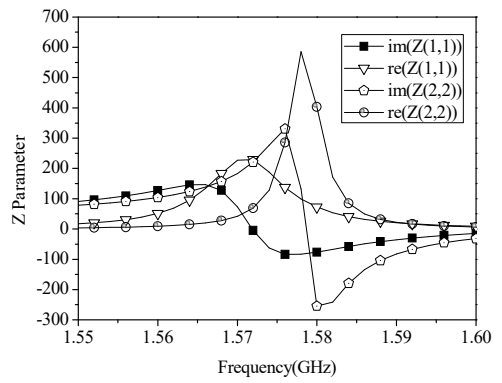

Figure 5. Comparison of the input impedance of dual port

\section{Adjustment of missile borne conformal antenna}

\subsection{Circular polarization performance adjustment}

In order to reduce the influence of conformation to the antenna and improve the circular polarization performance, the simulation on the width $W$, length $L$ and the size of the cut corner $c$ was carried. The results showed that the change of the above parameters cannot improve the circular polarization performance of the antenna when the microstrip patch remains square.

Then the simulation for the increment $d x$ is processed. The $d x$ is an increment added to $W$, which changes the square patch into rectangular patch. When $d x$ is increasing in a small area, the $S_{11}$ curve changes significantly, as shown in Figure 6(a). The minimum value of the antenna axis ratio decreases with the increase of $d x$, and the optimal frequency point moves to low frequency, which is optimal at $d x=0.4 \mathrm{~mm}$, as shown in Figure 6(b). The maximum value of the circularly polarized gain also moves to the low frequency, as shown in Figure 6(c)

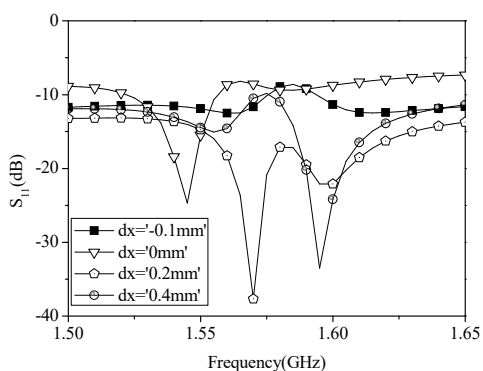

(a) Changes of $S_{11}$ with $d x$

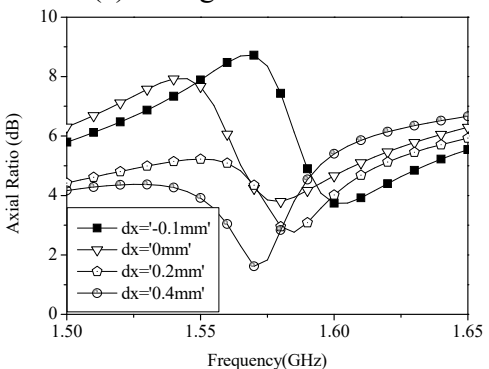

(b) Changes of axial ratio with $d x$

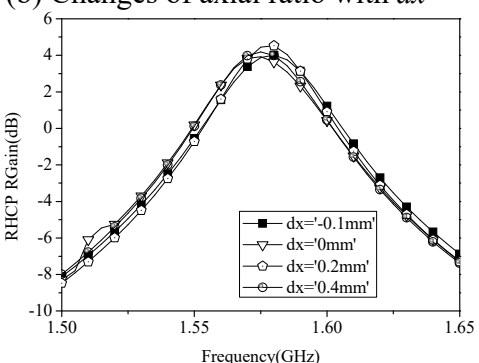

(c) Changes of circular polarization gain with $d x$

Figure 6. Change of antenna performance with $d x$

The change of $S_{11}$ and axial ratio in Figure 6 reflects the change of antenna input impedance. It can be seen that increasing the width $W$ can adjust the input impedance, reduce the impedance difference between the two ports, and improve the equal amplitude and orthogonality of the two signals. Therefore, we can adjust the width $W$ to reduce the conformal effect and improve the circular polarization radiation performance of the antenna. 


\subsection{Resonant frequency point adjustment}

When microstrip antennas are manufactured, the dielectric constant drift of the dielectric substrate will change the resonant frequency of the antenna. In this case, the antenna can be further adjusted by cutting corners. The antenna has four cutting corners, as shown in Figure 7. They are of the same size and are all isosceles right angled triangles, whose right angles are $c$ in length.

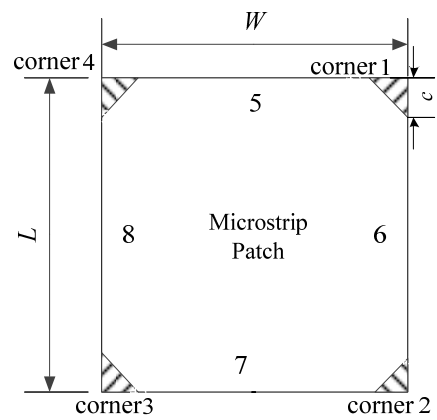

Figure 7. Diagram of the corner-cutting adjustment method of microstrip antenna

The $S_{11}$ curves, axial ratio curves, circular polarization gain curves and horizontal azimuth patterns of the antennas with different size of cutting corners are simulated, as shown in Figure 8. When the size of cutting corner increasing in a small range the antenna resonance frequency moves to high frequency, as shown in Figure 8(a). At the same time, the optimal frequency points of the axial ratio and the circular polarization gain of the antenna will also be shifted to the high frequency together, as shown in Figure 8(b) and 8(c). But the horizontal gain changes less, as shown in Figure 8(d). The resonant frequency can be adjusted by means of cutting corner, and the overall performance of the antenna is basically unaffected.

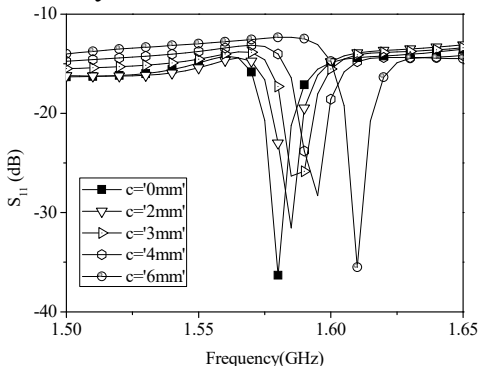

(a) $S_{11}$ curve

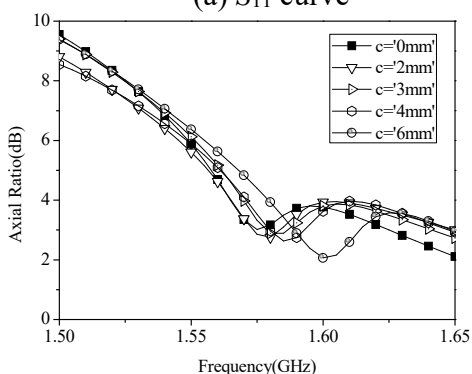

(b) Axial ratio curve

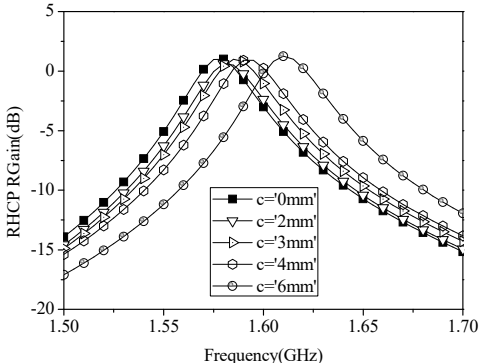

(c) Circular polarization gain curve

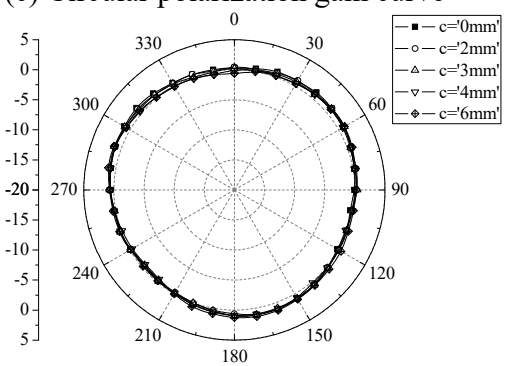

(d) Horizontal pattern

Figure 8. The change of the antenna performance with the size $c$ of the cut corner

\section{Antenna prototype performance test}

After adjustment and optimization, the final design scheme of the antenna is obtained. The size of the final antenna is $W=59.1 \mathrm{~mm}$ and $L=58.7 \mathrm{~mm}$, the rest design parameters remain unchanged. The missile- borne navigation antenna prototype is fabricated, as shown in Figure 9 .

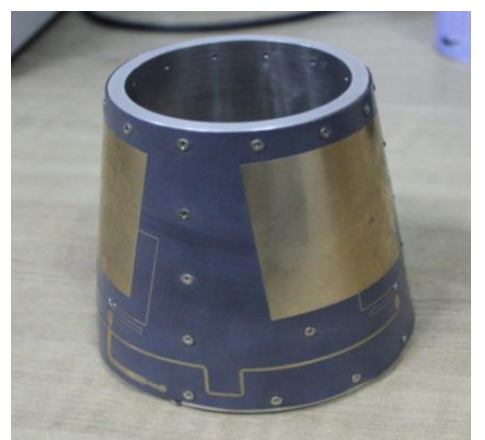

Figure 9. The missile-borne conformal navigation antenna prototype

The $S_{11}$ parameters, the circular polarization gain, the horizontal gain and the vertical gain of the antenna are tested. The measured data are compared with the HFSS simulation results. The $S_{11}$ curve of the antenna is shown as shown in Figure 10(a). In the range of $1.55 \sim 1.6 \mathrm{GHz}$, the antenna $S_{11}$ is less than $-10 \mathrm{~dB}$, and the working frequency band covers GPS L1 frequency point and Beidou navigation B1 frequency point. The $S_{11}$ curve of the antenna prototype is slightly lower than that of the simulation results. The circular polarization gain curve of the antenna is shown in Figure 10(b). The measured circular polarization gain is basically consistent with the simulated circular polarization gain.

The horizontal pattern and vertical pattern of the antenna at GPS L1 frequency and Beidou B1 frequency are measured, and the results are shown in Figure 10(c) and $10(\mathrm{~d})$. The maximum gain of antenna's horizontal gain at GPS L1 and Beidou B1 is $1.73 \mathrm{~dB}$ and $1.25 \mathrm{~dB}$ 
respectively, and the minimum gain is $-0.41 \mathrm{~dB}$ and $-0.9 \mathrm{~dB}$ respectively. Horizontal patterns at these two frequencies are basically circular and the out-of-roundness is $2.14 \mathrm{~dB}$ and $2.15 \mathrm{~dB}$ respectively. The antenna's vertical pattern is basically 8 -shaped, as shown in Figure 10(d)

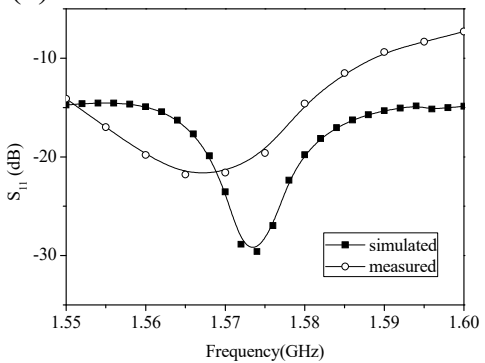

(a) $S_{11}$ curve

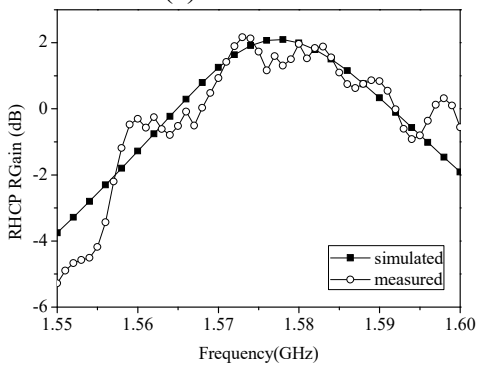

(b) Circular polarization gain curve

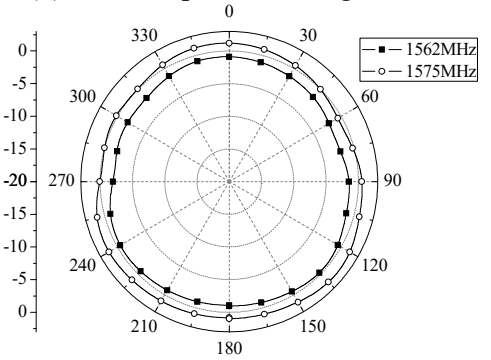

(c) Horizontal radiation pattern

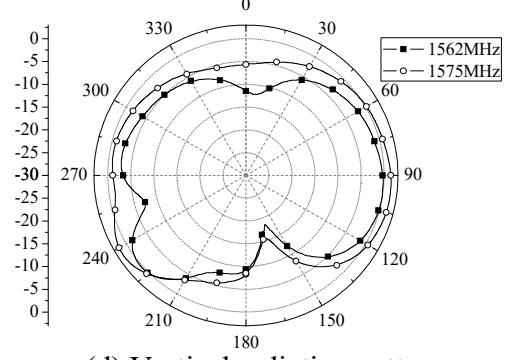

(d) Vertical radiation pattern

Figure 10. Comparison of antenna simulation results and measurement results

\section{Summary}

In this paper, a doubly fed circularly polarized conformal microstrip antenna array is designed and fabricated on electrically small missile body. In the design process, firstly, the influence of conformation on the performance of antenna element is simulated and analyzed. On this basis, the impedance matching is improved by adjusting the antenna width parameters, and the circular polarization performance of the conformal antenna is improved. Secondly, the method of cutting corner is used to adjust the resonant frequency of the antenna, which could facilitate the modification and adjustment of the antenna. Finally, according to the optimal design scheme, the antenna is fabricated and tested. The test results show that the $S_{11}$ parameter of the antenna is less than $-10 \mathrm{~dB}$ in the $1.55 \sim 1.60 \mathrm{GHz}$ frequency band. The maximum of the horizontal gain of the GPS L1 frequency point and the Beidou B1 frequency point is respectively $1.73 \mathrm{~dB}$ and $1.25 \mathrm{~dB}$. The horizontal patterns of the two frequency point are basically round, and the out-of-roundness is $2.14 \mathrm{~dB}$ and $2.15 \mathrm{~dB}$ respectively. The antenna has a good horizontal omnidirectional circular polarization radiation.

\section{Reference}

1. L. L. Wang, J. P. Liu. Journal of Microwaves, 33(S1), 63-67(2017).

2. X. D. Yang, B. Xu, Y. Bai, et al. Journal of Microwaves, 26(S2), 230-233(2010).

3. C. L. Li. Design of Microstrip Conformal Antenna Array (Xidian University, 2011).

4. J. Yang. Analysis and Research of Conformal Antenna Array on Thin Dielectric Carrier (University of Electronic Science and technology, 2011).

5. L. P. Hou. Analysis and Research of Conformal Micro- strip Antenna on Dielectric Carrier (University of Electronic Science and technology, 2009).

6. T. Liu, X. Y. Cao, J. J. Qin. Journal of Air Force Engineering University, 10(01), 79-82(2009).

7. S. J. Wang. Modern Electronics Technique, 34 (2011).

8. W. W. Yin. The Design and Simulation of Conformal An- tenna for Aircraft Carrier (Harbin Institute of Technology, 2015).

9. H. Y. Qi. Study of Experiment and Pattern Synthesis for Conformal Microstrip Arrays (National University of Defense Technology, 2010).

10. J. Ouyang. Analysis and Synthesis of Conformal Antenna and Array (University of Electronic Science and technology, 2008).

11. H. C. Liu. Research on Multi Beam Forming Technology of Conformal Antenna Array (Northwestern Polytechnical University, 2007).

12. F. W. Yao. Shipboard Electronic Countermeasure, 39(04), 34-36(2016).

13. H. P. Dou. Journal of North University of China, 38(02), 186-190(2017).

14. S. Y. Zheng, W. S. Chan, K. F. Man. IEEE: Microwave and Wireless Components Letters (2010).

15. W. Wang, J. G. Miao, B. Liang. Electronic Measurement Technology, 33(12), 1-3+28(2010). 